\title{
NOTA SOBRE NUEVAS LOCALIDADES DE POBLACIONES SILVESTRES DEL ZAPOTE PRIETO (DIOSPYROS XOLOCOTZII, EBENACEAE), ESPECIE AMENAZADA DEL OCCIDENTE DE MÉXICO
}

\author{
Ignacio TorRes ${ }^{1,3}$ y SANTIAgo Arizaga ${ }^{2}$
}

${ }^{1}$ Universidad Nacional Autónoma de México, Centro de Investigaciones en Ecosistemas, Laboratorio de Ecología y Evolución de Recursos Vegetales, Antigua Carretera a Pátzcuaro 8701, Colonia Ex-Hacienda de San José de la Huerta, 58190 Morelia, Michoacán, México.

${ }^{2}$ Universidad Nacional Autónoma de México, Escuela Nacional de Estudios Superiores Unidad Morelia, Antigua Carretera a Pátzcuaro 8701, Colonia ExHacienda de San José de la Huerta, 58190 Morelia, Michoacán, México.

${ }^{3}$ Autor para la correspondencia: itorresg@cieco.unam.mx

\section{RESUMEN}

Se documentan tres nuevas localidades del zapote prieto o aguacatillo (Diospyros xolocotzii Madrigal \& Rzedowski), una especie críticamente amenazada, así como su estructura poblacional y su hábitat. Dos de ellas son restos de poblaciones silvestres del zapote prieto en el estado de Michoacán. Una está representada por un solo individuo ubicado en el traspatio de una propiedad en Santa María de Guido. La otra está conformada por cinco árboles en una parcela agrícola de Santiago Undameo. Ambas localidades pertenecen al municipio de Morelia. Por el contrario, la tercera corresponde a la población silvestre más conservada hasta ahora conocida en el país de $D$. xolocotzii, la cual cuenta con alrededor de 1500 individuos. Se ubica hacia el norte de la cuenca del lago de Cuitzeo, dentro de una barranca en la localidad de San José de Las Pilas, municipio de Acámbaro, Guanajuato.

Palabras clave: aguacatillo, Cuitzeo, Diospyros, especie endémica, Guanajuato, Michoacán, población relicta.

\section{ABSTRACT}

Three new localities of Diospyros xolocotzii Madrigal \&Rzedowski (zapote prieto or aguacatillo), a critically endangered species, are recorded for Mexico, and a description of its 
population structure and habitat is presented. Two localities are relicts of wild populations of the zapote prieto in the state of Michoacán. One of them is represented by a single individual in the settlement of Santa María de Guido. The other one consists of five trees in an agricultural field in Santiago Undameo. Both localities belong to the municipality of Morelia, Michoacán.

In contrast, the third locality corresponds to the best conserved wild population of $D$. xolocotzii known until now in Mexico, which has about 1500 individuals. It is found in the northern part of the Cuitzeo lake basin, in a canyon in the locality of San José de Las Pilas, municipality of Acámbaro, in the state of Guanajuato.

Key words: aguacatillo, Cuitzeo, Diospyros, endemic species, Guanajuato, Michoacan, relict population.

\section{INTRODUCCIÓN}

Diospyros xolocotzii Madrigal \& Rzed. (Ebenaceae) fue descubierta en 1985 en el Manantial La Mintzita, en el municipio de Morelia, Michoacán. Se trata de un zapote silvestre comestible conocido como zapote prieto (Madrigal-Sánchez y Rzedowski, 1988). Diospyros xolocotzii es una planta arbórea dioica, caducifolia, la cual puede presentar uno o varios fustes y exhibe una alta reproducción vegetativa. Esta especie presenta una distribución reducida, de la cual solo se conocía una población conformada por 20 individuos en la localidad tipo.

El hábitat circundante a este manantial está sujeto a intensa perturbación y gran presión por cambio de uso de suelo, principalmente con fines agropecuarios y asentamientos humanos. Por estas y otras causas, parece que varios procesos biológicos esenciales para garantizar la supervivencia de la población (polinización, dispersión y reclutamiento) están siendo afectados negativamente. La fragmentación del hábitat ha determinado que los individuos de zapote queden confinados a montículos rocosos que rodean los cultivos y en menor proporción a la vegetación secundaria (Madrigal-Sánchez y Rzedowski, 1988; Sánchez-Atanacio, 2009).

En un trabajo reciente, Sánchez-Atanacio (2009) documentó para La Mintzita una población de 56 individuos de zapote prieto. Más de la mitad (55\%, es decir 31 ejemplares) son plantas juveniles aún no reproductivas, 29\% (16) corresponde a árboles masculinos y solo 16\% (9) a los femeninos. Debido a lo anterior, la Secretaría de Medio Ambiente y Recursos Naturales en la NOM-059 ha ubicado a D. xolocotzii como una especie amenazada (Anónimo, 2010).

En este trabajo se dan a conocer los resultados de una búsqueda sistematizada de nuevas localidades de D. xolocotzii en la cuenca del lago de Cuitzeo, así como 
su estructura poblacional y su hábitat, con la finalidad de actualizar su distribución geográfica como base para su conservación.

\section{MATERIALES Y MÉTODOS}

La prospección de nuevas poblaciones se llevó a cabo en la cuenca del lago de Cuitzeo dentro del matorral subtropical (Villa-Castillo, 1998). Durante el periodo de marzo de 2006 a diciembre de 2009, se realizaron un total de 39 salidas sistematizadas elegidas mediante una retícula de $5 \times 5 \mathrm{~km}$.

En los sitios donde se encontró al zapote se colectaron ejemplares en los periodos de floración y fructificación. La identificación se realizó con literatura especializada (Carranza, 2000), además de cotejar con ejemplares de los herbarios del Instituto de Ecología, A.C. (IEB) y de la Facultad de Biología de la Universidad Michoacana de San Nicolás de Hidalgo (EBUM). Los ejemplares herborizados se depositaron en los mismos herbarios y en el Centro de Investigaciones en Ecosistemas de la Universidad Nacional Autónoma de México - Campus Morelia. Para cada localidad se realizó una descripción del hábitat que comprendió: i) ubicación geográfica, ii) altitud, iii) tipo de vegetación según Rzedowski \& Calderón de Rzedowski (1987), iv) listado de especies arbóreas acompañantes y v) apreciación del estado de conservación de la población silvestre.

A partir del método descrito por Gentry (1988), se caracterizó su estructura poblacional en la localidad silvestre por medio de dos transectos de 50 x $5 \mathrm{~m}$ donde se registró: i) densidad, ii) sexo (presencia de flores o frutos), iii) altura del fuste, iv) altura total, v) número de ramas, vi) diámetro a la altura del pecho (DAP), vii) dos diámetros de copa y viii) presencia de daños de tipo antrópico clasificándola en dos categorías: evidencia de corte y marcas de fuego. Además se recorrió toda la extensión que comprende la población de la especie para tratar de establecer el total de individuos mediante conteo directo.

\section{RESULTADOS}

\section{Localidades de Michoacán}

Se encontraron dos nuevas localidades dentro del municipio de Morelia, una ubicada al sur y la otra al suroeste de la ciudad de Morelia (Cuadro 1). La prime- 
Cuadro 1. Descripción del hábitat de las nuevas localidades de D. xolocotzii y la localidad tipo.

\begin{tabular}{|c|c|c|c|c|c|c|}
\hline $\begin{array}{l}\text { Localidad } \\
\text { (municipio y } \\
\text { estado) }\end{array}$ & $\begin{array}{l}\text { Ubicación } \\
\text { geográfica }\end{array}$ & $\begin{array}{c}\text { Altitud } \\
\text { (m s.n.m.) }\end{array}$ & $\begin{array}{c}\text { Unidad } \\
\text { ambiental }\end{array}$ & $\begin{array}{c}\text { Estado de } \\
\text { conservación }\end{array}$ & $\begin{array}{l}\text { Número de } \\
\text { individuos }\end{array}$ & $\begin{array}{c}\text { Especies } \\
\text { arbóreas } \\
\text { acompañantes }\end{array}$ \\
\hline $\begin{array}{l}\text { San José de } \\
\text { Las Pilas, } \\
\text { Acámbaro, } \\
\text { Guanajuato }\end{array}$ & $\begin{array}{c}19^{\circ} 59^{\prime} 58^{\prime \prime} \mathrm{N} \\
100^{\circ} 53^{\prime} 32^{\prime \prime} \mathrm{W}\end{array}$ & 2077 & $\begin{array}{c}\text { matorral } \\
\text { subtropical }\end{array}$ & bueno & \pm 1500 & cerca de 16 \\
\hline $\begin{array}{l}\text { Santa María } \\
\text { de Guido, } \\
\text { Morelia, } \\
\text { Michoacán }\end{array}$ & $\begin{array}{c}19^{\circ} 40^{\prime} 16.6^{\prime \prime} \mathrm{N} \\
101^{\circ} 11^{\prime} 28.2^{\prime \prime} \mathrm{W}\end{array}$ & 2030 & $\begin{array}{c}\text { zona } \\
\text { urbana con } \\
\text { vestigios } \\
\text { de matorral } \\
\text { subtropical }\end{array}$ & $\begin{array}{l}\text { extremadamente } \\
\text { crítico }\end{array}$ & 1 & cerca de 4 \\
\hline $\begin{array}{l}\text { Santiago } \\
\text { Undameo, } \\
\text { Morelia, } \\
\text { Michoacán }\end{array}$ & $\begin{array}{c}19^{\circ} 35^{\prime} 44^{\prime \prime} \mathrm{N} \\
101^{\circ} 17^{\prime} 13^{\prime \prime} \mathrm{W}\end{array}$ & 2025 & $\begin{array}{l}\text { zona rural- } \\
\text { agrícola con } \\
\text { vestigios } \\
\text { de matorral } \\
\text { subtropical }\end{array}$ & muy crítico & 5 & cerca de 7 \\
\hline $\begin{array}{l}\text { ANP } \\
\text { Manantial } \\
\text { La Mintzita, } \\
\text { Morelia, } \\
\text { Michoacán }\end{array}$ & $\begin{array}{l}19^{\circ} 38^{\prime} 44.5^{\prime \prime} \mathrm{N} \\
01^{\circ} 16^{\prime} 28.4^{\prime \prime} \mathrm{W}\end{array}$ & 1900 & $\begin{array}{l}\text { zona rural- } \\
\text { agrícola con } \\
\text { vestigios } \\
\text { de matorral } \\
\text { subtropical }\end{array}$ & crítico & 56 & cerca de 16 \\
\hline
\end{tabular}

ra se encuentra en Santa María de Guido y está compuesta por un solo individuo masculino reproductivo de $9 \mathrm{~m}$ de altura, con diámetro promedio de copa de 3.78 $\mathrm{m}$, presenta un fuste principal y cuatro secundarios con un DAP promedio de 17.98 $\pm 9.21 \mathrm{~cm}$. El árbol crece en el patio de una vivienda dentro del área urbana de la comunidad.

La segunda localidad se ubica en Santiago Undameo y está conformada por cinco individuos que muestran una altura promedio de $6.50 \pm 1.18 \mathrm{~m}$, un diámetro promedio de copa de $7.35 \pm 1.73 \mathrm{~m}$ y un DAP promedio de $19.73 \pm 5.23 \mathrm{~cm}$. Cuatro de ellos se encuentran rodeando un terreno agrícola (dos femeninos, uno masculino y uno juvenil); el último es un árbol femenino de $7.98 \mathrm{~m}$ de altura y un diámetro de copa promedio de $9.25 \mathrm{~m}$. Está ubicado en el traspatio de una vivienda aledaña, a $590 \mathrm{~m}$ de distancia de los anteriores.

En ambas localidades aún es posible encontrar algunos vestigios de especies leñosas típicas del matorral subtropical, tales como Condalia velutina, Ipomoea murucoides, Forestiera phyllireoides, Casimiroa edulis, Erythrina sp., entre otras; sin 
embargo el estado de conservación que presenta D. xolocotzii es altamente crítico dada su baja densidad y ubicación.

\section{Localidad de Guanajuato}

Se encontró una sola localidad en una barranca cerca de la comunidad de San José de Las Pilas, que forma parte de la sierra de San Pablo Pejo, en el municipio de Acámbaro (Cuadro 1). Presenta una abrupta topografía con altitudes que oscilan de 1900 a $2570 \mathrm{~m}$.

La vegetación es un matorral subtropical con buen estado de conservación y abundantes especies leñosas caducifolias entre las que destacan: Acacia farnesiana, Casimiroa edulis, Cedrela dugesii, Celtis caudata, Condalia velutina, Ehretia latifolia, Erythrina sp., Euphorbia tanquahuete, Eysenhardtia polystachya, Ipomoea murucoides, Prosopis laevigata y Prunus brachybotrya. La presencia de Diospyros xolocotzii, conocido localmente como aguacatillo, es común; sin embargo tiene una distribución agregada.

Se encontró una densidad de $16.5 \pm 2.12$ individuos $/ 500 \mathrm{~m}^{2}$, con una altura promedio de $5.45 \pm 2.18 \mathrm{~m}$ que oscila desde $1.72 \mathrm{~m}$ hasta $11.50 \mathrm{~m}$. Presentan un diámetro promedio de copa de $3.95 \pm 1.88 \mathrm{~m}$ de diámetro, un fuste promedio de $2.02 \pm$ $0.91 \mathrm{~m}$ de altura, un DAP de $11.10 \pm 10.30 \mathrm{~cm}$ y con $5.21 \pm 3.59$ ramas por individuo.

La distribución de frecuencias de la altura es casi normal, con la mayor frecuencia de individuos (61\%) entre 3 y 6 m de altura. En contraparte, los árboles grandes (10.5-12 m) solo representan 6\%. El DAP mostró una distribución cargada hacia la izquierda, lo que sugiere que son más abundantes las plantas juveniles $(\leq 15$ cm de DAP; 79\% del total). Cabe destacar que en el área de muestreo no se encontró ninguna plántula de zapote prieto.

Esta población de D. xolocotzii presenta una gran cantidad de árboles reproductivos, al parecer dominada por individuos femeninos (Cuadro 1). Se estima la existencia de alrededor de 1500 plantas de ambos sexos. Por otro lado, hay numerosos indicios de daños por fuego y por machetazos.

\section{DISCUSIÓN}

Diospyros xolocotzii es una especie que, a pesar que recientemente se han encontrado más individuos en la localidad tipo (Sánchez-Atanacio, 2009), se había considerado endémica al Manantial La Minzita, Michoacán. En este trabajo primeramente se muestra que su distribución es más amplia de lo que se conocía, con la 
presencia de tres nuevos sitios, dos en Michoacán y uno en Guanajuato. SánchezAtanacio (2009) sugiere la existencia de más poblaciones dentro y fuera de la cuenca que comparten las mismas condiciones climáticas de La Mintzita en donde es factible hallarla. Es probable que nuevos esfuerzos de exploración revelen su presencia en lugares adicionales.

Otro aspecto importante es el avance en el conocimiento del estado de conservación de D. xolocotzii. En Michoacán, las localidades de Santa María de Guido y Santiago Undameo posiblemente representan vestigios de lo que se presume fueron poblaciones más grandes. Su estado de conservación es crítico, debido a diversos factores de disturbio causados por el hombre que han conllevado a las presuntas poblaciones silvestres a su extinción local, ya que la actual cantidad de individuos ( 1 y 5 , respectivamente) no es suficiente para asegurar la persistencia natural de la especie.

En La Mintzita la situación es muy similar, ya que el mantenimiento de la población de D. xolocotzii recae en tres árboles femeninos (Sánchez-Atanacio, 2009). Por lo tanto, de continuar las presiones generadas por el hombre que reducen la población y que afectan los procesos naturales de regeneración, en poco tiempo se podría extinguir localmente esta especie.

La propagación por semillas aún es factible para los árboles de la localidad de Santiago Undameo, ya que se observaron frutos fértiles. Sin embargo, no se apreciaron reclutamientos naturales. Posiblemente las condiciones ecológicas del suelo se han modificado negativamente como consecuencia de su intenso manejo con fines agrícolas. Por otro lado, se está construyendo una nueva vivienda en esta propiedad cerca de los zapotes, de manera que su destino es muy incierto.

En Santa María de Guido solo es posible la propagación vegetativa del único individuo que la conforma. No obstante, los propietarios demandan del espacio para ampliar su vivienda. Esto ha provocado que un árbol vegetativo de esta especie fuera talado en los dos últimos años.

Por el contrario, la población de Guanajuato es la que se encuentra en el mejor estado de conservación, con una estructura poblacional aún joven pero con suficientes individuos reproductivos de ambos sexos que garantizarían el reclutamiento de nuevas plántulas vía semillas. En nuestras visitas no encontramos evidencias de ello; sin embargo, tal situación puede deberse a un desfase de la producción de semillas. También es posible que nuestro muestreo se haya realizado en un año en que no hubo la suficiente lluvia como para evidenciar este reclutamiento en campo, o bien que los transectos se establecieron en sitios que no tienen las condiciones ideales para su crecimiento, ya que estaban relativamente cerca de una vereda transitada por los lugareños. Colateralmente, se aprecia la propagación vegetativa natural que 
posee la especie. Esta población presenta la mayor cantidad de individuos silvestres de $D$. xolocotzii hasta ahora conocida en el país, estimada en aproximadamente 1500 plantas (Cuadro 1).

No obstante que las poblaciones de Michoacán aquí registradas están prácticamente extintas, es urgente encaminar acciones para preservar los individuos existentes. Debido al tamaño que presentan, es difícil su trasplante; otra opción es mediante su propagación vegetativa. En este sentido es interesante comentar que Beltrán (2012) ha logrado injertar a D. xolocotzii. Por otro lado, es necesario reforzar la divulgación y educación ambiental entre el sector apropiado de la sociedad sobre el estado crítico que guarda esta especie con la finalidad de que cooperen a su conservación, al menos no dañándola.

\section{AGRADECIMIENTOS}

Agradecemos al Pas. en Ing. Forestal Román Bacilio López y a la M. en C. Isela Edith Zermeño Hernández, quienes colaboraron en el hallazgo de algunas localidades; al Dr. Jerzy Rzedowski por examinar parte del material botánico colectado; al Dr. Pablo Carrillo-Reyes, al Dr. Francisco Espinosa y al M. en C. Xavier Madrigal-Sánchez por la revisión y comentarios para mejorar el presente manuscrito; y finalmente a la familia Cesar Verduzco por las facilidades otorgadas para realizar este trabajo.

\section{LITERATURA CITADA}

Anónimo. 2010. Norma Oficial Mexicanas-059-Semarnat-2010. Protección ambientalEspecies nativas de México de flora y fauna silvestre-Categoría de riesgo y especificaciones para su inclusión, exclusión o cambio-Lista de especies en riesgo. Diario Oficial de la Federación. 30 de Diciembre de 2010. Segunda Sección. México, D.F., México.77 pp.

Beltrán, M. D. 2012. Evaluación del potencial de propagación vegetativa en zapote prieto (Diospyros xolocotzii). Tesis de licenciatura de Ingeniero Forestal. Instituto Tecnológico del Valle de Morelia. Morelia, Michoacán, México. 82 pp.

Carranza, E. 2000. Ebenaceae. Flora del Bajío y de Regiones Adyacentes 83: 1-9.

Gentry, A. H. 1988. Changes in plant community diversity and floristic composition on environmental and geographical gradients. Ann. Missouri Bot. Gard. 75: 1-34.

Madrigal-Sánchez, X. y J. Rzedowski.1988. Una especie nueva de Diospyros (Ebenaceae) del municipio de Morelia, estado de Michoacán, México. Acta Bot. Mex. 1: 3-6. 
Rzedowski J. y G. Calderón de Rzedowski. 1987. El bosque tropical caducifolio de la región mexicana del Bajío. Trace 12: 12-21.

Sánchez-Atanacio, F. 2009. Bases ecológicas y agronómicas del zapote prieto (Diospyros xolocotzii): una especie amenazada de Michoacán. Tesis de licenciatura en Agronomía. Instituto Tecnológico del Valle de Morelia. Morelia, Michoacán, México. 86 pp.

Villa-Castillo, B. 1998. Inventario de los recursos vegetales presentes en la cuenca del lago de Cuitzeo, Michoacán, México. Centro de Investigación y Desarrollo del Estado de Michoacán. Morelia, Michoacán, México. 110 pp. 